\title{
La Equidad de Género en Fe y Alegría Ecuador
}

\section{Antonio Narváez ${ }^{1}$ antoniodan41@gmail.com}

Artículo recibido en octubre 2016 y aprobado en noviembre 2016

\section{Resumen}

El presente ensayo se vincula a la experiencia del autor en el proceso de ir cayendo en la cuenta de un mundo que sistemáticamente vulnera la integridad de la mujer. La categoría "reconocimiento", conforme la comprende Paul Ricoeur en Caminos del Reconocimiento, es empleada para esclarecer la problemática del género en Fe y Alegría; en este sentido, en primer lugar, se pretende identificar las raíces y exponer las manifestaciones actuales de los conflictos vinculados a la invisibilización de la mujer por parte de la sociedad predominantemente patriarcal. En segundo lugar, se muestra lo que Fe y Alegría dice y hace en relación al género. De esta manera, se motiva a tomar una posición a favor de la promoción de la mujer y de la equidad de género en la institución.

Palabras clave: patriarcalismo, género, homogenización, invisibilización del género femenino.

\section{Abstract}

This essay derives largely from the author's experience to become aware of a world that systematically violates the integrity of women. The "recognition" category, as understood by Paul Ricoeur in "Caminos del Reconocimiento", is used to clarify the difficulties of gender in Fe y Alegría; in this sense, firstly, it is intended to identify the roots and present the current manifestations of the conflicts related to invisibilization of women by the predominantly patriarchal society. Second, it shows what Fe y Alegría says and does in relation to gender. In this way, it encourages to take a position in favor of the promotion of women and gender equity in the institution.

Keywords: patriarchy, gender, homogenization, invisibility of female gender.

1 Máster en Diseño y Gestión de Proyectos Socioeducativos (Universidad Central del Ecuador), Diplomado Superior en Investigación Social (CLACSO). Coordinador de Planificación y Gestión Estratégica de Fe y Alegría Ecuador. 


\section{Introducción}

La aproximación a la realidad de una persona siempre implica una gran dosis de respeto y sensibilidad, especialmente cuando atraviesa situaciones de dolor, injusticia o discriminación, como muchas veces sucede con las mujeres. Si bien se han dado grandes pasos a favor de la promoción de los derechos de la mujer, una mirada atenta revela que hay mucho por hacer para que los pueda ejercer y sea respetada en todos los ámbitos de la vida.

Esta tarea se vuelve difícil debido a que es indiscutible que la sociedad y cultura de la que todos y todas participamos es el principal lugar donde se reproducen conductas, comportamientos y posturas que no favorecen la promoción de los derechos a favor de la mujer, sino que por lo contrario, más fácilmente perpetúa actitudes que la discriminan.

El presente ensayo está vinculado a la experiencia del autor en su proceso de reconocimiento de un mundo que vulnera la integridad de las mujeres, y explicita los temas que esclarecen esta problemática en el lugar donde labora, esto es el Movimiento de Educación Popular Integral y Promoción Social Fe y Alegría. En este sentido, se posicionará el lugar epistemológico desde donde se revisarán algunas problemáticas de la mujer ecuatoriana. Posteriormente, se realizará una lectura de lo que Fe y Alegría dice y hace en relación al enfoque de género; de esta manera, se busca una toma de posición que permita bosquejar unas posibles líneas de acción que fomenten la equidad de género en la institución.

\section{Antecedentes de la propuesta}

\section{La investigación con enfoque de género}

En ¿Existe un método feminista? (Harding, 1987), la autora reflexiona en torno a las características de una investigación con enfoque de género, e indica que son tres los aspectos que deberían ser tomados en cuenta: la experiencia de vida, en cuanto que se rescata una vivencia 
propia, diferente de una realidad propia de los estudios andróginos, que no muestran las preocupaciones propias de las mujeres, ni su trayectoria de lucha ni de crítica ni de confrontación con el mundo masculino. La otra característica es el enfoque de los problemas femeninos propiamente, no los problemas del hombre blanco, occidental y burgués, que plantea preguntas que no afrontan las problemáticas de la mujer, ni sus intereses ni necesidades. Una última característica se refiere a que se presentan feminismos: feminismo de las latinoamericanas, de las europeas, etc., cada uno con características propias.

Al referirse a la integración de la mujer en los ámbitos intelectuales, la autora subraya que no basta únicamente con la participación de la mujer en círculos científicos masculinos porque en conjunto, se corre el riesgo de que se trabajen problemas planteados por hombres, lejos de las problemáticas propias de la mujer, además de que la misma aproximación al objeto de estudio contiene ya características andróginas que no consideran el aspecto relacional ni circunstancial entre el sujeto y el objeto estudiado, esto es lo que se conoce como la reflexividad de la ciencia.

La autora indica además, que es erróneo pensar que las experiencias científicas masculinas validan las femeninas, o lo contrario, pues se relativizarían unas a otras. Tampoco se podría afirmar que los hombres no pueden producir conocimiento en el campo feminista pues sería lo mismo que decir que un hombre de origen burgués está imposibilitado de aportar a la liberación de un pueblo. Importa ubicarse en la perspectiva feminista, evidenciar sus problemáticas propias y sus necesidades particulares, invisibilizadas por las posturas andróginas.

\section{Una experiencia frente al enfoque de género}

Al desarrollar las características de una investigación desde un punto de vista feminista, Sandra Harding (1987), es muy enfática al destacar la necesidad de que el autor de una investigación con enfoque de género debe explicitar su propia posición frente al problema que investiga. Esta postura va en la línea de que una investigación que busque 
descubrir las causas de una problemática social, deberá explicitar la posición del investigador frente a dicha problemática y explicitar el destino que ocuparán los resultados de la investigación (Katzer \& Samprón, 20112012).

Con esta premisa, es fundamental explicitar que el autor del presente ensayo es hombre, académicamente formado en una matriz patriarcal, pues los estudios secundarios los realizó en un colegio regentado por militares y su formación académica ha estado influenciada por una orden religiosa masculina de la cual formó parte. Entre los temas que le han llamado la atención siempre han estado las Ciencias Exactas, particularmente la Física, además la Lingüística, la Filosofía y la Teología. El interés por las problemáticas de género tiene raíces en la propia experiencia académica, donde descubrió que problemáticas semejantes pueden ser vividas diferentemente por hombres y mujeres.

En otros casos, el trabajo conjunto con mujeres lo llevó a confirmar la diferencia de sensibilidades entre hombres y mujeres; finalmente, fue muy esclarecedor caer en cuenta de la violencia contra las mujeres por el único hecho de pertenecer al género femenino, y que estos abusos son originados por varones; los acosos que sufren las mujeres en el transporte público, por poner un ejemplo, difícilmente lo experimentan los hombres.

Con el tiempo, el autor fue mirando, que en muchas disciplinas, son muy diferentes los estudios realizados por hombres y los realizados por las mujeres, y muy especialmente aquellos con enfoque de género, es decir, que no todo estudio realizado por una mujer evidencia la particularidad femenina; así, pueden existir estudios realizados por varones con enfoque de género que sí destaquen problemáticas propias que sufren las mujeres.

Una de las causas que origina los atentados a los derechos de la mujer está íntimamente relacionada con el fomento de la homogenización que promueve la globalización del mercado, además de la tendencia a invisibilizar las problemáticas sufridas por las mujeres, y que se evidencia, por ejemplo, en el desconocimiento del trabajo en los ambientes más domésticos o privados, mientras que destaca las labores 
realizadas en el ámbito público y por los hombres. Es justamente esta postura la que debe ser primeramente superada si se pretende aportar para que la mujer tenga un trato más justo y libre de violencia.

Con estos preámbulos, el autor se ubica con mayor conocimiento de causa ante una problemática que de suyo aparece prácticamente invisibilizada, pero a la vez sabe que la primera acción es una labor de concientización de sí mismo, de la organización a la que pertenece, que también es una obra eclesial, y de la misma mujer.

\section{El reconocimiento}

Un concepto muy útil que permite fundamentar el enfoque de género, es decir destacar el aporte de la mujer, fomentar sus derechos e inclusive las mismas luchas de reivindicación, es el "reconocimiento". Uno de los estudios más profundos y fundamentados sobre el reconocimiento es el desarrollado por Paul Ricoeur, Caminos del reconocimiento (2005). Inicia con una aproximación lexicográfica donde articula las definiciones alrededor de identificación y distinción: para identificar hay que distinguir y para distinguir se necesita identificar. Al referirse a la identificación, explica que comprende tanto a la identidad como a la ipseidad, sea de una persona o de un objeto.

Otro aspecto destacado en el reconocimiento, proviene de la conjugación de su verbo en la voz pasiva: ser reconocido, lo cual permite vincular con la lucha por el reconocimiento que Hegel desarrolla en la Fenomenología y que en la actualidad sustenta los debates acerca de identidad y diferencia, así como las exigencias para el reconocimiento de posturas éticas, políticas o sociales que personas o grupos humanos los exigen (Fraser y Honneth, 2006).

\section{¿Por qué la subordinación de la mujer?}

En la profundización sobre los motivos que llevan a la invisibilización de la mujer, y su consecuente subordinación al hombre, Sherry B. Ortner desarrolla el artículo " ¿Es la mujer con respecto al hombre lo que 
la naturaleza con respecto a la cultura?", destaca que la violencia contra la mujer es un hecho presente en prácticamente todas las culturas y en casi todos los tiempos. En este sentido, la autora se pregunta por el motivo de esta realidad. En la respuesta, se remite a la condición natural de la mujer y del hombre. Como en muchas especies, el macho se encarga de la protección y la mujer a la maternidad, perpetuando así la especie. En el caso humano, esta situación generaría una situación de comodidad a la mujer. Sin embargo no se tienen estudios antropológicos serios en este sentido, de manera que esta hipótesis estaría descartada.

Por otra parte, la autora se pregunta ¿existe algo negativo propio de la naturaleza que se asocie a la mujer?, en otras palabras, ¿hay algo del ámbito no humano vinculado a la mujer que, como consecuencia, genera esta percepción de subordinación? En la respuesta a esta cuestión, se muestra que en el afán por subyugar la naturaleza, la humanidad desarrolla la cultura como una forma de dominio de la naturaleza; mediante los ritos y símbolos procura purificar, sanear y ejercer el dominio sobre las fuerzas naturales.

La autora se inclina a pensar que la mujer ha sido asociada a la naturaleza, mientras que los hombres a la cultura, entendiéndola como mediación para dominar la naturaleza. La autora dice no tanto asociación sino la consideración de la mujer como más cercana a la naturaleza y el hombre más cercano a la cultura. Es decir los hombres trascienden la naturaleza mientras las mujeres no. Entre los motivos que aparentemente explicarían esta subordinación, destaca el propio cuerpo de la mujer que la situaría más cercana a los procesos de la naturaleza, mientras que el del hombre lo dejan más libre para los culturales; por otra parte, siendo el cuerpo la mujer más favorable al cuidado de los recién nacidos y la familia, se asocia a roles sociales considerados menos trascendentes que los culturales, configurando, finalmente, una siquis que le llevarían a asumir esta posición de subordinación.

La supuesta cercanía de la mujer a la naturaleza y del hombre a la cultura, es por sí misma, una construcción cultural, pues tanto hombres como mujeres están en contacto con la naturaleza, aunque la sicología, el cuerpo y el rol social de la mujer, parecen jugar en su contra. La cercanía de la mujer a la naturaleza y por tanto su estatus de inferioridad 
en relación al hombre se institucionaliza, lo que implica una subordinación en funciones y participación.

La superación del carácter inferior de la mujer respecto del hombre, debería ser, consecuentemente, trabajada tanto a nivel institucional como a nivel cultural: al mismo tiempo que se procuran leyes para una participación política, laboral, académica, entre hombres y mujeres, de forma equitativa, se debería trabajar el aspecto cultural, simbólico y lingüístico que vinculan a la mujer a procesos más cercanos a la naturaleza que a los trascendentes, rituales o culturales.

\section{Un contexto de injusticias contra la mujer}

\section{Violencia de género}

Las mujeres que asisten a Fe y Alegría pertenecen a familias con muchas necesidades. Muchas de ellas son maltratadas cada día por sus esposos o parejas. Hacen parte del $60 \%$ de mujeres que reciben algún tipo de maltrato, sea sicológico, físico, sexual o de patrimonio. Muchas de ellas callan porque aprendieron que "aunque pegue y mate, marido es". Han sido humilladas, menospreciadas, encerradas, vigiladas o amenazadas con quitarles a sus hijos o correrles de la casa.

Lo más impresionante es que el $90 \%$ de la mujeres que ha sufrido algún tipo de violencia por parte de su pareja no se ha separado. De este grupo el $54 \%$ no piensa separarse, el $23,5 \%$ se separó por algún tiempo y regresó con su pareja y el 11,9\% piensa en separarse.

Según la Encuesta Nacional de Relaciones familiares y violencia de Género contra las Mujeres (INEC, 2012), el 52,5\% de las mujeres (a pesar de ser sujeto de violencia) no se separa porque consideran que "las parejas deben superar las dificultades y mantenerse unidas", el $46,5 \%$ piensa que "los problemas no son tan graves" y el 40,4\% "quiere a su pareja", mientras el $22 \%$ "no se puede sostener económicamente". 
De acuerdo a la etnia, el porcentaje más alto que ha vivido algún tipo de violencia se concentra en la población indígena con el 67,8\% seguida de la mujer afro-ecuatoriana con el $66,7 \%$.

\section{Discriminación laboral}

En cuanto a la situación laboral, entre marzo del 2014 y marzo del 2015, la tasa de empleo global aumenta en 0,51 puntos porcentuales para las mujeres y 1,39 puntos porcentuales para los hombres, de manera que la tasa de empleo global para las mujeres es 1,78 puntos porcentuales menor que la de los hombres.

En el mismo período la tasa de empleo adecuado aumenta en 0,47 puntos porcentuales para los hombres y disminuye en 4,67 puntos porcentuales en las mujeres, de manera que las mujeres tienen 16.24 puntos porcentuales menor que la de los hombres.

La tasa de empleo inadecuado aumenta en 0,74 puntos porcentuales para los hombres y aumenta en 4,99 puntos porcentuales en las mujeres, de manera que la tasa de empleo inadecuado para las mujeres es 14,4 puntos porcentuales mayor que la de los hombres.

En cuanto al desempleo, disminuye en 1,36\% para los hombres y 0,55 puntos porcentuales para las mujeres, de manera que el desempleo en las mujeres el 1,78 puntos porcentuales mayor en las mujeres que los hombres.

En cuanto al salario, mientras el hombre con empleo tiene un salario promedio de 483,13 USD, la mujer con empleo es de 398,83 USD.

Por último, las mujeres tienen un promedio laboral de 36 horas semanales, los hombres tienen 36 horas. (Censos, 2015).

La empresa Deloitte en Ecuador, solo 30\% de las empresas consultadas asegura contar con algunas políticas de flexibilidad para madres ejecutivas. De estas, las empresas del sector financiero son las que más políticas presentan para beneficiar a las madres. 
Las posiciones gerenciales también tienen diferencias sustanciales marcadas por género. El estudio de Deloitte revela que solo un $13 \%$ de empresas tiene una mujer en el cargo de regenta general y un $3 \%$ en cargo de presidenta.

Esta realidad no solo deja a las mujeres en desventaja en cuanto a los niveles de salario, sino que además podría ser perjudicial para las empresas.

La firma McKinsey\&Co realizó el año pasado un estudio en Londres con unas 366 empresas y evidenció que en aquellas donde existía un mayor balance de género en cargos de liderazgo había una mayor probabilidad de presentar resultados financieros superiores al promedio nacional del sector.

Las principales áreas donde se destacan las mujeres ejecutivas, según el estudio de Deloitte, son los departamentos de finanzas (24\%) y recursos humanos (26\%).

Si clasificamos a las mujeres por nivel de instrucción, el 70\% de las que asisten a centros de alfabetización ha sufrido algún tipo de violencia y aunque las que más educación tienen sufren menos violencia, los porcentajes no bajan del $50 \%$.

\section{La Legislación ecuatoriana y los derechos de la mujer}

En la constitución de 1998, se dieron algunos avances, especialmente garantizaba el derecho a la integridad personal y a una vida libre de violencia, a la igualdad ante la ley y la no-discriminación. En cuanto a la participación política, fomenta la equidad de mujeres y hombres en cuanto a los procesos de elección popular, instancias de dirección y decisión en el ámbito público, en la administración de justicia, los organismos de control y los partidos. También garantizaba el derecho de las mujeres a tomar decisiones libres y responsables sobre su vida sexual y reproductiva. Reconoce de manera formal el trabajo doméstico como labor productiva así como la igualdad y corresponsabilidad en la familia y el apoyo a las jefas de hogar, además de la obligatoriedad del Estado de aprobar políticas públicas e institucionalidad para impulsar la igualdad de las mujeres. 
La constitución de Montecristi (2008) incluye el principio de equidad de género entre hombres y mujeres en los ámbitos de la salud, la educación, el trabajo, el empleo, la comunicación, la producción, la familia, la participación, entre otros, y garantiza el ejercicio de cada uno de esos derechos en igualdad. Los derechos sexuales y reproductivos, la valoración y distribución igualitaria del trabajo doméstico y las actividades de autosustento, el derecho a una vida libre de violencia que incluye la garantía de cada persona a la integridad física, psicológica y sexual, constituyen derechos en sí mismos pero también son preceptos a observarse al momento de ejercer otros.

Esta constitución brinda protección especial y prioritaria a niños, niñas, adolescentes, mujeres adultas, mujeres embarazadas y en período de lactancia, adultas mayores y desplazadas, sobre todo cuando éstas sean víctimas de maltrato, violencia doméstica o sexual; y/o explotación sexual. Otorga el derecho a la seguridad social a quienes tienen a su cargo el trabajo no remunerado en el hogar, en su mayoría mujeres; y se determina la prioridad de dotar de vivienda a mujeres jefas de hogar.

En resumen, la constitución del 2008 acerca a los ecuatorianos y ecuatorianas a vivir en una sociedad igualitaria, pero su vigencia no será posible solamente con la Constitución, es necesario entenderla, interpretarla, difundirla, impulsarla, vigilar su cumplimiento, legislar acorde con sus mandatos, ejercer el derecho a la participación que otorga, generar jurisprudencia. Las mujeres tienen en ello una gran tarea por desarrollar.

Relativamente hace pocos años, en 1995, el sistema legal asume como delito la violencia contra la mujer (Ley 103). Se especifica más la diferencia entre delito y contravención en temas de violencia contra la mujer o integrantes del núcleo familiar, en el Código Orgánico Integral Penal (COIP), vigente desde el 10 de agosto del 2014, el maltrato dentro de la pareja y en el núcleo familiar por golpes, insultos, acoso y abuso sexual, así como las humillaciones, tienen sanciones penales porque son delitos. Antes del COIP estaba en vigor la Ley Contra la Violencia a la Mujer y la Familia, constituida en la denominada Ley $N^{\circ} 103$. En esta los casos de violencia contra la mujer eran una contravención y 
conocidos en las comisarías, que después fueron reemplazadas por las unidades de violencia intrafamiliar.

La tipificación de delitos contra la mujer, como el femicidio, fue una propuesta que nació de Fiscalía, en noviembre del 2011, en los primeros meses de iniciada la administración de Galo Chiriboga Zambrano, fiscal General del Estado. Como resultado de largas jornadas de trabajo se elaboró una propuesta para incluir delitos de violencia de género basados en las relaciones dominantes y de poder hombre-mujer, documento que se presentó ante la Comisión de Justicia de la Asamblea Nacional.

Es delito cuando los actos violentos se repiten una y otra vez, con conocimiento del daño que se genera en la víctima, con toda la gana de hacerlo. El agresor se aprovecha de su posición de poder y de la vulnerabilidad de la persona afectada que puede ser una mujer, un niño, una adolescente o una persona de la tercera edad.

Sus victimarios: padre, madre, esposo, esposa, tío, tía... todo aquel que por su rango jerárquico dentro del núcleo familiar tenga autoridad y poder sobre la víctima. La violencia se tipifica en el COIP como física (Art. 156), sexual (Art. 158) y psicológica (Art. 157). Sus sanciones varían según el caso y sus agravantes, pueden ser de meses y también de años de penas privativas de la libertad.

La menos visible, porque no deja huellas como hematomas (moretones), pero igual de destructiva porque destroza el sentido de ser humano, es la violencia psicológica. Esta es considerada como golpes directos a la autoestima a través de insultos, amenazas, humillaciones, desprecios o privación autoritaria de su libertad.

Se trata de una contravención cuando la agresión a la víctima no es grave, es decir, que el daño ocasionado no dura más de tres días. En el Art. 159 del COIP se determina la sanción de pena privativa de libertad de siete a 30 días si las lesiones causadas le provocan incapacidad de hasta tres días.

La incapacidad se determina por la falta de dinamismo o de energía que la persona tiene para hacer sus actividades diarias como consecuencia de lo sucedido o cuando necesita descansar para regresar a su vida normal. 
Estos casos se resuelven en las Unidades Judiciales de la Niñez y la Familia, en los juzgados de contravenciones.

\section{El género en Fe y Alegría Ecuador}

Una vez desarrollados los preámbulos como claves de lectura para interpretar la problemática de la equidad de género, se procurará contrastarlos con la equidad de género en Fe y Alegría Ecuador, lo que implica desarrollar estas características a partir de una aproximación a la bibliografía de la institución y a proyectos que involucran a la mayoría de actores y actoras que hacen Fe y Alegría, de manera que se puedan pensar acciones en favor de la equidad de género.

\section{Participación de la mujer en el nacimiento de Fe y Alegría}

Para todos y todas que hacen parte de Fe y Alegría, es conocido que este Movimiento "no nació de y progresó por la fácil confianza en las chequeras bien provistas" sino que fue la respuesta natural de una mirada apostólica, movida por la compasión cristiana, a una realidad marcada por la miseria, la injusticia y la exclusión. Si bien su fundador, el P. José María Vélaz nutrió su fe y su compromiso en el manantial de la espiritualidad ignaciana y heredó el tesón y la garra de los grandes misioneros jesuitas, casi nunca se revisa la historia de Fe y Alegría desde la mirada de las mujeres que participaron en su fundación.

Por una parte, las estudiantes de la universidad Católica Andrés Bello, reciben la invitación de uno de los directores espirituales de la universidad. Conocerán el rostro empobrecido de Caracas. Allí se dedican a preparar para la catequesis a las niñas y niños del barrio. Esta realidad las golpea sobremanera, no conocían sino sus hogares acomodados donde todo lo tenían a pedir de boca. Con sus compañeros y el capellán se emocionan ante la idea de crear una escuela para aquellos niños y niñas que fuera más allá de las catequesis; con las mujeres del barrio apoyan la propuesta. Otra mujer, Patricia, habitante del barrio, con 8 hijos y un marido que trabaja como albañil, lo anima para ofrecer la casa que acaban de construir y se eduquen los primeros estudiantes. 
Otras jóvenes que están a punto de terminar el bachillerato se ofrecen como maestras y más adelante se unirán algunas religiosas que desde entonces no han dejado Fe y Alegría.

Muchas de estas mujeres deben sortear dificultades; a menudo son molestadas por los hombres del barrio que las acosan. No conciben que puedan hacer algo diferente que servir en el hogar, a los hijos o a los hermanos menores, pues les recuerdan que su lugar es la casa.

Así nace Fe y Alegría, que en un lapso de diez años iniciará su expansión a Ecuador y a otros países de América Latina. En nuestro país, la idea de fundar Fe y Alegría emociona a muchas religiosas y se crean los primeros centros educativos; una de las primeras secretarias ejecutivas, será Blanca Crespo, que colaboró con todo lo que pudo en los primeros años de Fe y Alegría Ecuador. Muchas jóvenes y niñas participarán años más tarde en la famosa rifa de Fe y Alegría.

\section{En los documentos institucionales}

Los valores inspiradores de la identidad de Fe y Alegría se explicitan más directamente en algunos de los documentos que recogen su ser y quehacer: el Ideario y los Congresos Internacionales. Además se considerará Las Políticas Federativas de la Equidad de Género, y el decreto 14 de la Congregación General 34 de la Compañía de Jesús, que es la orden religiosa encargada de Fe y Alegría.

En el Ideario, documento que condensa la razón de ser del Movimiento, Fe y Alegría define como un Movimiento de Educación Popular que nacido e impulsado por la vivencia de la Fe Cristiana, frente a situaciones de injusticia, se compromete con el proceso histórico de los sectores populares en la construcción de una sociedad justa y fraterna. Entre los objetivos que persigue se encuentra una referencia a las mujeres, en cuanto que se plantea "promover la formación de hombres y mujeres nuevos, conscientes de sus potencialidades y de la realidad que los rodea, abiertos a la transcendencia, agentes de cambio y protagonistas de su propio desarrollo." (Carrasco, 2008: 11). Este documento se elaboró en el XV y XVI Congreso Internacional de Fe y Alegría, en 1984 y 1985. 
En los Congresos Internacionales hay dos referencias a la equidad de género, en el XXX Congreso Internacional, en 1999, "Educación y tecnología para un desarrollo sustentable y demandas del mundo del trabajo", en la primera parte del documento de trabajo (con el mismo nombre del Congreso) en la explicitación del contexto mundial, visto como un mundo donde los procesos sociales se imponen desde una perspectiva cada vez más global y menos humana, trayendo consigo un cambio vertiginoso en los ámbitos económico, cultural y político. Es en el ámbito político donde se verifica una "crisis ideológica generalizada y un fortalecimiento de tendencias autoritarias en los gobiernos; la universalización del discurso sobre principios en materia de derechos humanos, equidad de género y solidaridad hacia los más pobres (Carrasco: 2008: 157).

Otra referencia se encuentra en el XXXV Congreso Internacional "Fe y Alegría: actor internacional y agente de sensibilización para la transformación social" (2004), en la cuarta parte del documento final, que se refiere a los criterios y orientaciones para el trabajo de sensibilización y acción pública, explícitamente en la defensa de la justicia y equidad, no solo desde la perspectiva económica y social, sino "incorporando la equidad de género (para garantizar el acceso de la mujer a los derechos y beneficios sociales, en igualdad de condiciones, y promover su participación en la sociedad, con liderazgo e identidad propios)" (Carrasco, 2008: 312).

Parece importante destacar que el XXXVII Congreso Internacional, "Educación y promoción social comunitaria" (2006), en la tercera parte del documento final, "Criterios para la promoción social", la explicación de la constitución de la persona como sujeto, pide la transformación de mapas y diccionarios mentales, y explica que la identidad de género no es equivalente a la identidad sexual, sino una "convención sociocultural". Afirma que la identidad sexual se construye sobre la realidad biológica de manera que la identidad de género es socialmente construida a partir de la diferenciación biológica. Es más, afirma que el sujeto es masculino o es femenino, de la manera cómo se construya socialmente esa identidad. Aclara que como son identidades relacionales, dependerá también de ese proceso de ser hombre y mujer las relaciones de equidad o de subordinación entre personas y al interior de la sociedad (FIFYA, 2008: 384). 
En el III Plan Estratégico de la Federación Internacional de Fe y Alegría 2010-2014, se encuentra una significativa referencia a la equidad de género, en la justificación del plan estratégico, al reconocer las debilidades del contexto interno, particularmente cuando admite que "todavía no se tiene una comprensión plena del enfoque de equidad de género en los programas" especialmente los relativos a la educación y promoción social (Fe y Alegría, 2009: 28, 78).

En el breve decreto 14 de la Congregación General de la Compañía de Jesús, si bien no se habla de equidad de género, pasa revista a la situación de la mujer en la sociedad y la reconoce particularmente discriminada por el varón. Aunque no dice que los jesuitas participan de esta discriminación, al menos podrían no haberla distinguido. La reacción de la Compañía consiste en reconocer una 'feminización de la pobreza' y un 'rostro femenino de la opresión', ante lo cual y movidos por su carisma originario, corporativa e individualmente se comprometen a superarla. Entre las mediaciones a emplearse, se destacan: la enseñanza explícita, en todos los apostolados, de la igualdad esencial entre la mujer y el varón; el apoyo de los movimientos de liberación de la mujer, y la promoción de su participación en la vida pública; la atención particular al fenómeno de la violencia contra la mujer; el uso del lenguaje inclusivo; la promoción de la educación de la mujer y, en particular, la eliminación de toda forma de discriminación injustificada entre muchachos y muchachas en el proceso educativo.

En la Política de Género de la Federación Internacional de Fe y Alegría (2011), se busca proporcionar un marco de acción en el campo del género para el conjunto de las Fe y Alegría. El objetivo es construir colectivamente justicia, con perspectiva de equidad de género, y desde la visión de desarrollo humano integral sustentable para todas las personas, tanto en la gestión institucional del Movimiento Fe y Alegría como en su acción con los sectores poblacionales con quienes se relaciona para hacer realidad su Misión. El principal fundamento es la afirmación de la dignidad humana, igual y absoluta tanto para el hombre como para la mujer. Las políticas son las siguientes:

1. Construir e institucionalizar el enfoque de Equidad de Género en Desarrollo Humano Integral Sustentable de Fe y Alegría. 
2. Integrar el enfoque de Equidad de Género en Desarrollo Humano Integral Sustentable tanto en las políticas, como en la praxis, y la acción pública de Fe y Alegría.

3. Transversalizar y fortalecer la equidad de género en toda la acción y las proyecciones de Fe y Alegría.

4. Fomentar el empoderamiento individual y colectivo, desde la perspectiva de equidad de género, en el Movimiento de Educación Popular y Promoción Social Fe y Alegría

En el Plan Estratégico 2015-2019 de Fe y Alegría Ecuador, la misión dice: "Soñamos con un Ecuador donde todas y todos tengamos las mismas posibilidades de una educación para la vida, transformadora, liberadora, inclusiva y de calidad, con una Fe y Alegría más profética, que responda a los nuevos contextos y necesidades educativas, con una presencia e incidencia política renovada en las nuevas fronteras de exclusión."

Además, uno de los valores de Fe y Alegría es la "equidad de género", de manera que "la educación ha de partir del reconocimiento de cada ser humano en su particularidad esencial y, por tal razón, se hace imprescindible un trabajo que busque la igualdad de derechos y oportunidades de las personas, más allá de los roles previamente definidos culturalmente en relación al sexo.

\section{Actoras y Actores del proceso educativo de Fe y Alegría}

Las y los principales actores del proceso educativo de Fe y Alegría son niñas, niños, jóvenes, docentes y directivos/as.

En general, al referirse a las y los actores, fuera de un lenguaje inclusivo, la institución parece no reconocer la diversidad de género. Por ejemplo, en las Memorias 2011 (Narváez, 2012) en las estadísticas se mencionan únicamente valores totales de estudiantes, sin indicar cuántos son niñas, cuántos niños, jóvenes, maestras, maestros.

En otros espacios formativos, como el MIFA (Movimiento Infantil Fe y Alegría), el CEFA (Campamentos Ecuatorianos Fe y Alegría), no 
se verifica la perspectiva de género en ninguno de los módulos formativos. Algo parecido se puede afirmar de los proyectos educativos o pastorales destinados a la formación de las y los docentes, no hay ninguno que hable explícitamente de la equidad de género (Narváez, 2013). Esto no quiere decir que no se haga nada en favor de la equidad de género. Eventualmente se ofrecen cursos sobre enfoque de género. Ahora bien, ningún espacio formativo tiene el peso de otros programas más sistemáticos.

En cuanto a los directivos, se observa una tendencia a la equidad de género, la dirección está en manos de un hombre y la subdirección está a cargo una mujer. De las seis regionales, cuatro están lideradas por hombres y dos por mujeres. Las cuatro áreas institucionales: pastoral, educación, acción pública y administración, tres están a cargo de mujeres y una a cargo de un hombre.

Sin embargo de la relativa equidad directiva, desde una perspectiva simbólico - cultural se puede observar que de una u otra manera, Fe y Alegría encarga ciertos procesos de mayor profundidad a los hombres, mientras que lo logístico tiende a ser asignado a la mujer.

\section{Conclusiones y Recomendaciones}

Desde un punto de vista legal, se puede decir que Fe y Alegría tiene garantizado el marco jurídico para promover y fortalecer la equidad de género.

Desde una mirada del reconocimiento, se puede observar un relativo avance. Si en el año 1984, que se formuló el Ideario, este no se expresa en un lenguaje inclusivo, no obstante reconoce a la mujer como sujeto de explotación en el contexto de otros/as marginados/as, explotados/as y excluidos/as, en el año 2011 se formula la Política de género de la Federación Internacional de Fe y Alegría, que procura afrontar la explotación y la discriminación contra la mujer. Esto se confirma en la práctica, pues al menos en el lenguaje se busca distinguir lo masculino de lo femenino. 
Desde la misma perspectiva del reconocimiento, se puede verificar que aún falta por explicitar y describir el rostro femenino de la pobreza, de la opresión y la exclusión. Desde una perspectiva despatriarcalizadora, se podría leer que Fe y Alegría no ha sido lo suficientemente determinante en identificar la subordinación de la mujer, no obstante promueve la transformación, la liberación y la construcción de la historia. Esta suposición se podría fortalecer aún más, si se considera que el tema ha sido escasamente considerado en los Congresos Internacionales, y ninguno de ellos se ha dedicado a la equidad de género; más aún si el propio plan estratégico de la Federación reconoce que el tema no ha sido suficientemente comprendido. Mirando los espacios formativos del movimiento, también se descubre la invisibilización de la problemática del género al no incluirse la perspectiva de género.

Como discursos a partir de los cuales se pueden inspirar acciones, está el decreto 14 de la Congregación General 34 de la Compañía de Jesús y la Política de género de la Federación Internacional. Desde todas las perspectivas, aunque corto, el Decreto 14 parece más profundo en su análisis y sus propuestas: reconoce la invisibilización de la problemática, denuncia la inequidad de género y propone acciones en la educación, la sociedad, la cultura, el lenguaje. El segundo documento muestra que aún se está construyendo una perspectiva de equidad de género y podría ser más arriesgado en el análisis y la propuesta, no obstante no coarta las posibilidades de acción a favor de la equidad de género.

En general, se constata que institucionalmente hay apertura al fortalecimiento del enfoque de equidad de género. De hecho, el último Plan estratégico asume como un valor la equidad de género.

Por otra parte, se observa que hay mucho por trabajar, en cuanto al conocimiento del sentido y de las implicaciones de un enfoque de género en la institución. Si bien el plan estratégico lo tiene como uno de sus valores, resta incorporarlo en los procesos institucionales, ya sean pedagógicos, pastorales, inclusive en la elaboración de los proyectos educativos. 


\section{Referencias}

Asamblea Constituyente, (2008). Constitución del Ecuador. Versión digital.

Carrasco, Luis (Compilador), (2008). El pensamiento de Fe y Alegría. Documentos de los Congresos Internacionales 1984-2007, Caracas, Federación Internacional Fe y Alegría.

Chávez, Patricia, "Estado, descolonización y patriarcado", en: Chávez, P. et alii, Despatriarcalizar para descolonizar la acción pública, La Paz, Vicepresidencia del estado Plurinacional de Bolivia. Versión digital: https://www.vicepresidencia.gob.bo/IMG/pdf/depatriarcalizacion_rev.pdf.

Compañía de Jesús, Decreto 14 de la Congregación General 34 de la Compañía de Jesús, versión digital, http://www.jesuitas.info/Document/documentos.htm.

Deloitte. (2015). Mujeres 2015: una radiografía de las ejecutivas ecuatorianas. Versión digital: https://www2.deloitte.com/content/dam/ Deloitte/ec/Documents/deloitte-analytics/Estudios/Estudio\%20 Mujeres\%202015.pdf.

Tapia, E. (2015) "La mujer enfrenta la desventaja laboral". En: El Comercio, $1^{\circ}$ de mayo de 2015 . Enlace: http://www.elcomercio.com/actualidad/mujeres-desventaja-laboral-hombres.html.

Federación Internacional de Fe y Alegría, (2009). III Plan Estratégico de la Federación Internacional de Fe y Alegría 2010 - 2014. Federación Internacional Fe y Alegría.

Federación Internacional de Fe y Alegría, (2011). Política de Género de la Federación Internacional de Fe y Alegría.

Fraser, N., Honneth, A. (2006). ¿Redistribución o reconocimiento? Un debate político filosófico, Madrid, Ediciones Morata.

Harding, Sandra. (1987). ¿Existe un método feminista? Versión digital: http://148.206.107.15/biblioteca_digital/capitulos/81-2350ske.pdf. 
Instituto Nacional de Estadísticas y Censos (Inec). (Marzo, 2015). Encuesta Nacional de Empleo, Desempleo y Subempleo. Indicadores Laborales. Versión digital: http://www.ecuadorencifras.gob. ec/documentos/web-inec/EMPLEO/2015/Marzo-2015/Presentacion_Empleo_Marzo_2015.pdf.

Instituto Nacional de Estadísticas y Censos (INEC). Secretaría Nacional de Planificación y Desarrollo (SENPLADES), (2012). Primera Encuesta Nacional de Relaciones Familiares y Violencia de Género contra las Mujeres. Versión Digital: file:///C:/Users/Antonio/ Downloads/violencia\%20genero\%20desayuno\%20(1).pdf.

Katzer, L., Samprón, A. "El trabajo de campo como proceso. La "etnografía colaborativa" como perspectiva analítica". En: Revista Latinoamericana de Metodología de la Investigación Social. N². Año 1. Oct. 2011 - Marzo 2012. Págs.: 59-70.

Narváez, Antonio, (2010). El respeto activo: del reconocimiento a la valoración. (Disertación previa a la obtención del título de licenciado en Filosofía), Quito, PUCE.

Fe y Alegría. (2013). Fe y Alegría. Memorias 2011-2012, Quito, Fe y Alegría Ecuador. Versión digital: _ http://www.feyalegria.org.ec/images/biblioteca/memorias/Memoria\%20Institucional\%20Fe\%20 y\%20Alegr\%C3\%ADa\%202011-2012.pdf.

Fe y Alegría. (2015). Plan estratégico 2015-2019. Quito. Fe y Alegría Ecuador.

Ortner, Sherry, "¿Es la mujer con respecto al hombre lo que la naturaleza con respecto a la cultura?", Perú, Biblioteca virtual de Ciencias Sociales. Versión digital.

Presidencia de la República (DEL POZO, Hugo, Dir.), (2011). Ley Orgánica de Educación Intercultural. Quito. Registro Oficial. Versión digital.

Ricoeur, Paul (2005). Caminos del reconocimiento, Madrid, Trotta. 